\title{
PPP2R5D-Related Intellectual Disability as a Novel Cause of Amyoplasia
}

\author{
Brian Wahlig ${ }^{1}$, Kiley Poppino ${ }^{2}$, Carol Wise ${ }^{2}$, and Steven Sparagana ${ }^{3}$ \\ ${ }^{1}$ University of Texas Southwestern Medical School \\ ${ }^{2}$ Texas Scottish Rite Hospital for Children \\ ${ }^{3}$ University of Texas Southwestern Department of Neurology and Neurotherapeutics
}

August 28, 2020

\begin{abstract}
Amyoplasia is the most common form of arthrogryposis, a congenital condition characterized by multiple joint contractures. Current literature on amyoplasia suggests it is a sporadic condition without a genetic basis. We describe a patient with the musculoskeletal characteristics of amyoplasia and an underlying rare and possibly causal genetic variant.
\end{abstract}

\section{Key Clinical Message}

Amyoplasia may be associated with genetic variations including the rare condition PPP2R5D-related intellectual disability.

\section{Introduction}

Arthrogryposis is a complex musculoskeletal condition defined as multiple congenital contractures affecting two or more parts of the body. ${ }^{1}$ The overall prevalence of arthrogryposis is estimated to be 1:3000 live births. ${ }^{2}$ Amyoplasia is a specific cause of arthrogryposis that represents roughly one third of all cases. ${ }^{2}$ The classic presentation of amyoplasia includes adducted and internally rotated shoulders, extended elbows, flexed wrists, and frequently, clubfeet. ${ }^{3}$ Amyoplasia has long been considered to be a sporadic condition due to fetal akinesia in utero. Until recently, no chromosomal or single gene variants have been linked to this diagnosis. ${ }^{4}$ We present a unique case of a patient presenting with the musculoskeletal features of amyoplasia who was found to have a gene variant causing PPP2R5D-related intellectual disability (MIM 616355), a rare genetic condition associated with neurodevelopmental delay, speech impairment, and macrocephaly. ${ }^{5}$

\section{Case Presentation}

The child is a 3-year-old white male born via cesarean section at 35 weeks gestation for uterine fibroids and polyhydramnios. The child had been diagnosed prenatally with clubfeet noted on fetal ultrasound. At birth, the patient experienced respiratory distress and poor feeding necessitating a month-long hospitalization and gastrostomy tube placement. The infant was noted to have multiple joint contractures, hypotonia, and macrocephaly which prompted an initial genetic workup shortly after birth. This workup yielded a normal chromosome karyotype, chromosome microarray analysis, and FISH for trisomies 13, 18, and 21. Additionally, testing was negative for myotonic dystrophy and spinal muscle atrophy 1 and 2. Workup for additional anomalies included ultrasound of the head and abdomen, brain MRI, and echocardiogram, and all were normal for the child's age. Ophthalmology evaluation at birth was only significant for mild to moderate microphthalmia. Family history was negative for neuromuscular conditions or arthrogryposis.

The child presented to a pediatric orthopedic surgeon at 7 weeks of age. Physical exam was consistent with a diagnosis of amyoplasia. He had adducted and internally rotated shoulders, extended elbows, and wrist 
flexion contractures with no active motion in his fingers. He also had bilateral thumb-in-palm deformities. In the lower limbs, he had stiff hips with limited abduction and severe bilateral clubfeet scoring 20 out of 20 on the Dimeglio scale. His knees had full range of motion. Pelvic ultrasound and subsequent pelvic x-ray demonstrated both hips were reduced.

Initially, the child's orthopedic treatment regimen consisted of stretching the upper limbs and feet. Later, hand splinting as well as Ponseti casting for clubfeet was implemented. He underwent 3 rounds of Ponseti casting with weekly cast changes, but his care was interrupted by a two weeklong hospitalization due to viral gastroenteritis. Subsequently, he underwent two additional rounds of Ponseti casting before undergoing bilateral open tendo Achilles tenotomies to treat his residual equinus deformity. Concurrently, he underwent a left extensor carpi ulnaris to extensor carpi radialis brevis tendon transfer and volar fascia release to address his left-hand deformity. Postoperative upper and lower limb casts were applied for four weeks. At four weeks follow up, the patient was transitioned to upper limb splints and ankle-foot-orthoses, with parental instructions to begin range of motion exercises. At that time, he was able to dorsiflex both feet to neutral and had improved wrist and thumb positioning on the left side.

Throughout this period of orthopedic treatment, the patient was also followed by the neurology service. Neurology ordered a whole exome sequencing (WES) analysis which revealed a de novo heterozygous c.598G $>$ A (p.Glu200Lys) variant in the PPP2R5D gene. Additionally, there were variants of unknown significance in 3 genes linked to similar clinical phenotypes. These included a hemizygous variant in the CLCN4 gene as well as heterozygous variants inFBN2 and PYROXD1. However, in all three of these variants of unknown significance, one of the parents is an asymptomatic heterozygous carrier of the same variant. Additional testing revealed a normal baseline EEG and repeat brain MRI at age one year and five months demonstrated a paucity of white matter volume in the frontal, parietal, and anterior temporal lobes, as well as dilation of the ventricular system that appeared to be due to ex-vacuo change, and a persistent cavum septum vergae. A small foramen magnum and upper cervical canal was also noted but no impingement of neural elements was appreciated. Recently, he was diagnosed clinically with focal seizures with altered awareness (despite normal EEGs).

\section{Discussion}

The PPP2R5D gene encodes the protein B56 which is an isoform of the regulatory subunit of protein phosphatase $2 \mathrm{~A}(\mathrm{PP} 2 \mathrm{~A}){ }^{6}{ }^{6}$ This particular isoform is highly expressed in the brain and is believed to be involved in cell growth, gene transcription, and other key cellular processes. ${ }^{7}$ PPP2R5D-related intellectual disability is a rare disorder caused by mutations to this gene that is characterized by mild to severe neurodevelopmental delay, speech impairment, and macrocephaly. ${ }^{5}$ Other features that may be present include: autism spectrum disorder, seizures, ophthalmologic abnormalities, and hypotonia. ${ }^{5}$ This patient demonstrated several of these characteristics including speech impairment, macrocephaly, seizures, and hypotonia but has not to date been diagnosed with autism or any ophthalmologic abnormalities. Brain MRI findings in PPP2R5Drelated intellectual disability are nonspecific. However, this child does possess brain findings that had been previously reported in association with PPP2R5D-related intellectual disability including ventricular dilation (4 patients), white matter abnormalities ( 1 patient), and cavum vergae ( 1 patient). ${ }^{6,8}$ To date, only 23 patients with an average age of 9.3 years (range 0.3 - 53 years) have been reported in the literature with PPP2R5D-related intellectual disability. ${ }^{6,8-10}$ Musculoskeletal involvement in these patients is rare but has been described. Of the 23 patients previously reported, 3 had scoliosis, 1 had hip dysplasia, 1 had $3^{\text {rd }} / 4^{\text {th }}$ finger syndactyly, 1 had camptodactyly of the $4^{\text {th }}$ toe, and 1 had scoliosis and hip dysplasia. ${ }^{6,8-10}$ This child did not present with any of these previously reported musculoskeletal findings. Instead, he possessed the features of amyoplasia including adducted and internally rotated shoulders, extended elbows, flexed wrists, and clubfeet. Additionally, while orthopedic treatment in these patients has not been explicitly described, this patient's upper limbs and feet have been treated with a combination of physical therapy, casting, splinting, and surgery - as described. To our knowledge, no patient with PPP2R5D-related intellectual disability has been concomitantly found to have joint contractures typical of amyoplasia.

Arthrogryposis is a descriptive term rather than one specific diagnosis. An estimated 400 unique diagnoses 
are believed to lead to the features of arthrogryposis. ${ }^{11}$ It is important to identify the underlying cause of arthrogryposis when possible as this can aid in the implementation of appropriate treatment. ${ }^{1}$ For example, it is beneficial to identify those with amyoplasia as these patients typically have average to above average intelligence which gives them the opportunity for productive and independent lives if their orthopedic functionality is maximized. ${ }^{4}$ While several of the distal arthrogryposes have been associated with genetic abnormalities, amyoplasia, until recently, was not classically linked with any genetic mutations. ${ }^{4,11}$ One recent report linked complete absence of skeletal muscle (i.e. segmental amyoplasia) in distal arthrogryposis type 1 patients with mutations in the MYLPF gene. ${ }^{12}$ In another case, a patient was described with the classic musculoskeletal features of amyoplasia as well as Leigh syndrome, a genetic condition causing mitochondrial dysfunction, related in his case to heterozygous variants inSURF1 ${ }^{13}$

The child in this case report possesses a c.598G >A (p.Glu200Lys) variant in the PPP2R5D gene. This variant has been previously described as a cause of PPP2R5D-related intellectual disability. ${ }^{5}$ While this patient's variant was de novo and not present in either parent, it is not possible to completely exclude the possibility of parental germline mosaicism. Therefore, the recurrence risk of PPP2R5D-related intellectual disability to siblings is estimated to be $1 \% .^{5}$ This is important in the genetic counseling of his parents, as amyoplasia has historically been considered to be sporadic without recurrence in subsequent offspring. ${ }^{4}$ Additionally, while this patient does possess variants of unknown significance in other genes related to their clinical phenotype (including a mutation in FBN2, the gene associated with Distal Arthrogryposis Type $9)^{14}$ in each case, one of the asymptomatic parents possesses the same variant making it highly unlikely these represent pathological mutations. Similarly, though this patient could theoretically possess two co-existing diagnoses, we consider this unlikely given the extensive clinical and genetic evaluations.

\section{Conclusion}

This case highlights the importance of genetic evaluation in patients presenting with atypical arthrogryposis. Additionally, it expands the list of conditions that can present with arthrogrypotic features. Furthermore, this is the second case reported of a patient with the classic musculoskeletal features of amyoplasia presenting with an underlying genetic abnormality, calling into question the traditional understanding that amyoplasia is a non-genetic condition. ${ }^{13}$ Continued vigilance of individuals with arthrogryposis may prompt identification of additional causative conditions, and possibly, additional genetic causes of amyoplasia.

\section{Authorship List}

BW researched the patient's underlying condition and drafted the manuscript. KP contributed to the drafting of the manuscript. CW provided insight into the underlying genetic condition and contributed to editing of the manuscript. SS provided insight into the patient's neurological features and contributed to editing of the manuscript.

Acknowledgement: We would like to thank the providers in the orthopedic surgery and neurology departments at Scottish Rite for Children. We would like to specifically acknowledge Dr. Karl Rathjen for his guidance and for providing clinical care to this patient.

Conflicts of Interest: The authors declare no conflicts of interest.

\section{References}

1. Bamshad M, Van Heest AE, Pleasure D. Arthrogryposis: a review and update. The Journal of bone and joint surgery American volume.2009;91 Suppl 4:40-46.

2. Hall JG. Arthrogryposis multiplex congenita: etiology, genetics, classification, diagnostic approach, and general aspects. J Pediatr Orthop B. 1997;6(3):159-166.

3. Hall JG, Reed SD, Driscoll EP. Part I. Amyoplasia: a common, sporadic condition with congenital contractures. Am J Med Genet.1983;15(4):571-590.

4. Hall JG, Aldinger KA, Tanaka KI. Amyoplasia revisited. Am J Med Genet A. 2014;164a(3):700-730. 
5. Mirzaa G, Foss K, Nattakom M, Chung WK. PPP2R5D-Related Neurodevelopmental Disorder. In: Adam MP, Ardinger HH, Pagon RA, et al., eds. GeneReviews $((R))$. Seattle (WA): University of Washington, SeattleUniversity of Washington, Seattle. GeneReviews is a registered trademark of the University of Washington, Seattle. All rights reserved.; 2019.

6. Houge G, Haesen D, Vissers LE, et al. B56delta-related protein phosphatase 2A dysfunction identified in patients with intellectual disability. J Clin Invest. 2015;125(8):3051-3062.

7. Biswas D, Cary W, Nolta JA. PPP2R5D-Related Intellectual Disability and Neurodevelopmental Delay: A Review of the Current Understanding of the Genetics and Biochemical Basis of the Disorder. Int J Mol Sci. 2020;21(4).

8. Shang L, Henderson LB, Cho MT, et al. De novo missense variants in PPP2R5D are associated with intellectual disability, macrocephaly, hypotonia, and autism. Neurogenetics. 2016;17(1):43-49.

9. Loveday C, Tatton-Brown K, Clarke M, et al. Mutations in the PP2A regulatory subunit B family genes PPP2R5B, PPP2R5C and PPP2R5D cause human overgrowth. Hum Mol Genet. 2015;24(17):4775-4779.

10. Yeung KS, Tso WWY, Ip JJK, et al. Identification of mutations in the PI3K-AKT-mTOR signalling pathway in patients with macrocephaly and developmental delay and/or autism. Mol Autism. 2017;8:66.

11. Hall JG, Kimber E, van Bosse HJP. Genetics and Classifications.J Pediatr Orthop. 2017;37 Suppl 1:S4-s8.

12. Chong JX, Talbot JC, Teets EM, et al. Mutations in MYLPF cause a novel segmental amyoplasia that manifests as distal arthrogryposis. Am J Hum Genet. 2020;107(2):293-310.

13. Wilnai Y, Seaver LH, Enns GM. Atypical amyoplasia congenita in an infant with Leigh syndrome: a mitochondrial cause of severe contractures? Am J Med Genet A. 2012;158a(9):2353-2357.

14. Tsipouras P, Del Mastro R, Sarfarazi M, et al. Genetic linkage of the Marfan syndrome, ectopia lentis, and congenital contractural arachnodactyly to the fibrillin genes on chromosomes 15 and 5 . The International Marfan Syndrome Collaborative Study. N Engl J Med.1992;326(14):905-909. 